\title{
New insight into the mechanisms of lung metastasis
}

Metastatic bladder cancer is associated with a very poor prognosis-the survival rate at 5 years is less than $10 \%$. New research into how bladder cancer spreads to the lung, reported by Dan Theodorescu and colleagues in the Journal of Clinical Investigation, might pave the way for new treatment strategies to prevent metastasis.

Back in 2002, Theodorescu and his group reported their discovery of RhoGDI2 (RhoGTP dissociation inhibitor 2) as a suppressor of metastasis in human bladder cancer cell lines. As well as demonstrating that it could inhibit experimental lung metastasis, they also found that RhoGDI2 expression in patients with bladder cancer was inversely proportional to survival.

In this new study, researchers set out to investigate the mechanics of how RhoGDI2 exerts its suppressive effect using a variety of complementary pharmacological and genetic models in vivo and in vitro. First, they found a strong negative correlation between the expression of RhoGDI2 and versican (VCAN), a chemoattractant and component of the extracellular matrix. Accordingly, high expression of VCAN was found to be associated with poor prognosis in patients with bladder cancer.

Further experiments revealed that VCAN has a critical role in metastatic colonization of the lung, attracting macrophages from bone marrow to the metastatic site. During the early stages of metastasis, macrophages provide support

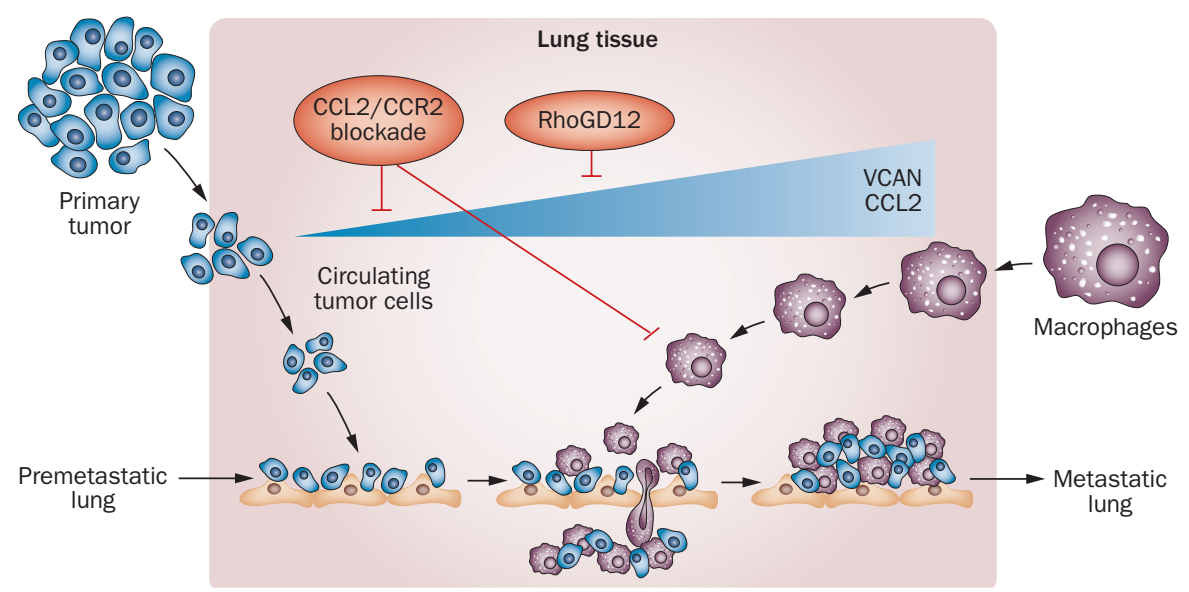

to cancer cells, allowing them to gain a foothold in the lung and promoting their growth into a clinically significant tumor.

The team's key finding, however, was that RhoGDI2 is responsible for downregulating VCAN expression, thus suppressing macrophage infiltration and preventing the growth and survival of cancer cells in the lung. "This is a novel mechanism of metastasis suppression in bladder cancer," says Theodorescu. "RhoGDI2 harnesses the microenvironment and the immune system in a way that might be utilized therapeutically."

Researchers found that the ability of VCAN to attract macrophages depends on the chemokine CCL2, and drugs that inhibit its receptor (CCR2) are already in clinical trials for other conditions. Theodorescu told Nature Reviews Urology that he is now establishing a clinical trial of CCR2 inhibitors in combination with endothelin inhibitors in patients with bladder cancer. Previous data have also implicated endothelin signaling downstream of RhoGDI2 in this setting.

Theodorescu is quick to point out that such a treatment strategy would only be feasible in the adjuvant setting, when patients have no detectable metastasis but might harbor micrometastases. Macrophages are probably much less important in the maintenance of established metastatic tumors than in tumor development.

Sarah Payton

Original article Said, N. et al. RhoGDI2 suppresses lung
metastasis in mice by reducing tumor versican expression
and macrophage infiltration. J. Clin. Invest. doi:10.1172/
JCI61392

